\title{
Stochastic Resonance and Multi-modal Firing Patterns in Single-neuron Models
}

\author{
Danny Linders \\ Bert Kappen \\ Department of Medical Physics and Biophysics, University of Nijmegen \\ $\mathrm{RWCP}^{*}$ Novel Functions SNN ${ }^{\dagger}$ Laboratory \\ Nijmegen, The Netherlands
}

\begin{abstract}
In analyzing data from experiments on periodically stimulated neurons, the interspike interval histogram shows a multi-modal distribution and under certain conditions, stochastic resonance. We discuss a simple integrate and fire model of a single neuron which is capable of describing these two features. As input for the neuron we use a periodic signal combined with poisson noise, this gives results that resemble data from experiments.
\end{abstract}

\section{Introduction}

There has been a great number of experiments in the past involving measurements on single neurons in the presence of some external stimulus. In the special case of a periodic and excitatory stimulus a typical phenomenon is observed: the neuron of interest tends to fire with the same period as the period of the stimulus it receives. We call this the coherent signal.

It depends on the strength of the stimulus whether a spike occurs every cycle or not. In the latter case we observe multi-modality in interspike interval histograms (ISIHs): peaks at integer multiples of the driving period.

Changing the noise level at the input of the neuron gives a different picture of the IsIH. At a specific noise level a maximum occurs in the coherent power at the output of the neuron (this is the power of the coherent signal, see above). This is called stochastic resonance (SR).

It was back in 1960 that Gerstein (Gerstein and Kiang, 1960) observed a multimodal ISIH when they discussed several ways to analyze data from experiments on single neurons. They used anesthetized cats whom they presented an auditory stimulus, being clicks at various rates and intensities. Siegel (Siegel, 1990) did similar experiments with light instead of sound. Sensory experiments on monkeys were done by Mountcastle (Mountcastle et al., 1969). In all these experiments the phenomenon of multi-modal firing patterns was observed. This effect was discussed by Gerstein and Mandelbrot (Gerstein and Mandelbrot, 1964). They represented the neuron by a random walk model, with a reflecting

\footnotetext{
*Real World Computing Partnership
}

$\dagger^{\dagger}$ Foundation for Neural Networks 
barrier at a resting potential and an absorbing barrier at the threshold. Adding a drift term, the strength of which was periodically varied in time: they obtained ISIHs like the ones found in the experiments of Gerstein and Kiang.

Douglas (Douglass et al., 1993), too, observed multi-modal IsIHs in experiments on the mechanoreceptors of the crayfish. In addition, he observed SR in varying the noise level of the stimulus. (Although he used a slightly different definition of SR than we will discuss below.) This phenomenon was discussed by Longtin (Longtin, 1993). He treated SR in two models: a double well potential and an excitable neuron model following the Fitzhugh-Nagumo equations.

Our goal is to demonstrate that $\mathrm{SR}$ can be observed in a neuron model with much more simple dynamics, but still biologically plausible.

\section{The Model}

A suitable model seems to be an integrate and fire model in which the soma potential $v(t)$ plays a central role. Initially the potential starts at zero. It is influenced by two inputs and a decay. The neuron input is divided in two parts: the noise and the signal. The noise is modeled as poisson distributed spikes with ar average interval $\tau_{\text {noise }}$ of constant strength $\epsilon_{\text {noise }}$, while the signal has a stcady period $\tau_{s i g}$ and strength $\epsilon_{s i g}$. The potential is increased by the noise and the signal and decreased at a constant rate by the decay with strength $\gamma$. The change in soma potential can thus be written as

$$
\dot{v}(t)=\epsilon_{s i s} \sum_{k=1}^{\infty} \delta\left(t-k \tau_{s i, y}\right)+\epsilon_{\text {nois } \epsilon} \xi\left(\frac{t}{\tau_{\text {noise }}}\right)-\gamma,
$$

where $\xi($.$) denotes a poisson process. At the time the potential reaches a$ threshold $\theta$, the neuron fires and the potential resets to zero.

The choice of a linear decay as an approximation of an exponential decay is motivated by our aim to find an analytical expression for the SR in this model. In the case of exponential decay (and ncise from a standard Wiener process) the model turns into an Ornstein-Uhlenbeck process, for which no expression can be found for first passage times. This means that one cannot calculate the probability of firing at a specific time after the last

input will only make things more complicated, thus this model is better left behind. In the model we describe here, if no signal is applied, it is possible to write down the probability that no spike has occurred during a time interval $0 \leq t \leq \tau$. The following resuit is due to Pyke (Pyke, 1959).

$$
\begin{aligned}
P\left\{\sup _{0 \leq t \leq \tau}(v(t)) \leq \theta\right\}=e^{-\tau / \tau_{n o \text { ioe }}} & \sum_{n=0}^{\lfloor\gamma \tau+\theta\rfloor} \frac{\gamma \tau+\theta-n}{n !}\left(\gamma \tau_{n o i s e}\right)^{-n} \\
& \times \sum_{j=0}^{\lfloor\theta\rfloor}\left(\begin{array}{c}
n \\
j
\end{array}\right)(j-\theta)^{j}(\gamma \tau+\theta-j)^{n-j-1}
\end{aligned}
$$

For notational convenierce this formula has been written for the case the process has been scaled so that $\epsilon_{\text {roise }}$ equals one, thus $\gamma \rightarrow \gamma / \epsilon_{\text {noise }}$ and $\theta \rightarrow 0 / \epsilon_{\text {noise }}$. 


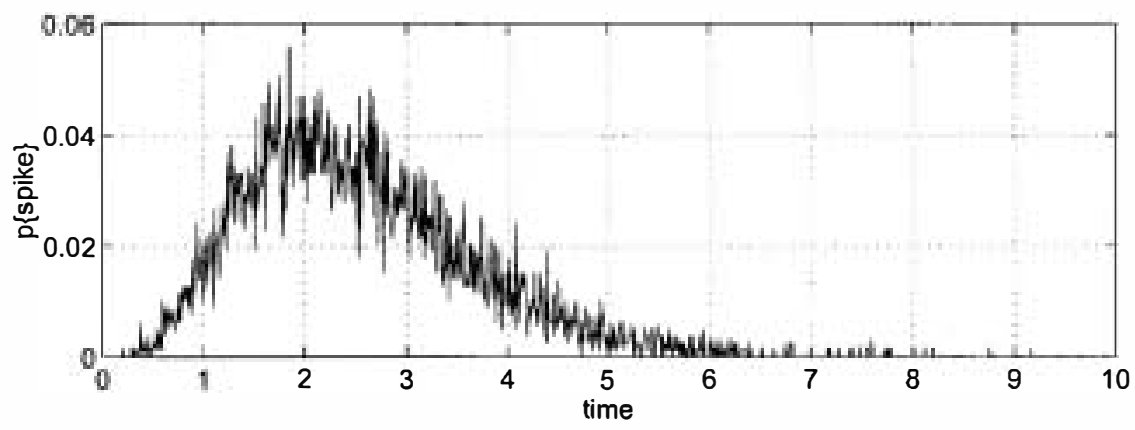

Figure 1: The ISIH of spontaneous activity. Parameters $0=4, \gamma=0.05$, $\tau_{\text {noise }}=0.5$ and $\epsilon_{\text {noise }}=1$.

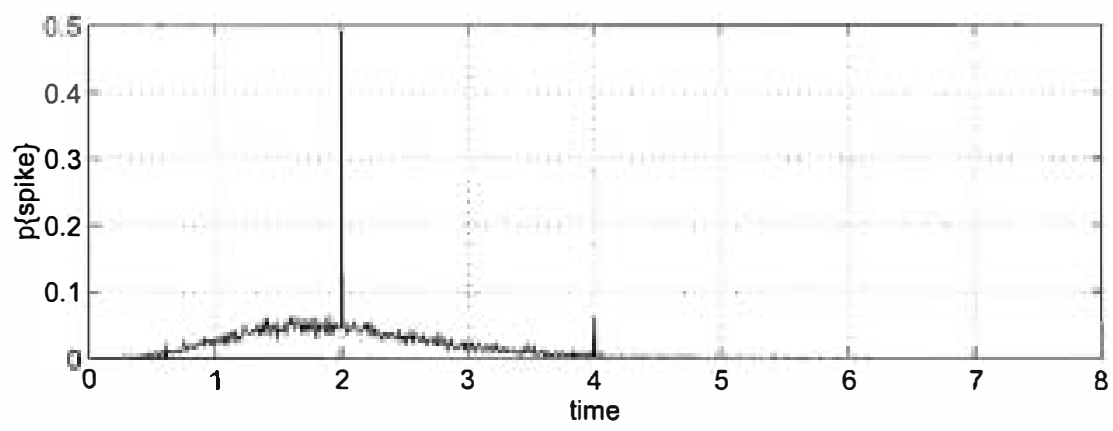

Figure 2: The multi-modal ISIH with a driving force. Parameters $\theta=4, \gamma=$ $0.05, \tau_{\text {sig }}=2, \epsilon_{\text {sig }}=0.7, \tau_{\text {noise }}=0.5$ and $\epsilon_{\text {noise }}=1$.

\section{Results}

First we treat the case that no signal is given to the neuron, only noise. The output the neuron then produces, can be viewed as spontaneous activity. Whether the input noise must be seen as a collection of random inputs from other neurons or as the result of unknown processes in the neuron itself, or perhaps both, is not argued. The ISIH then looks like Fig. 1.

Adding a periodic and excitatory stimulus makes the occurrence of a spike at the moment of the stimulus more probable. This results in peaks at integer multiples of the driving period. The multi-modal ISIH is plotted in Fig. 2.

The first peak in this figure represents spikes that have occurred with the same frequency as the stimulus. We called this the coherent part of the output signal. By varying the noise level we can observe a change in the height of this peak: from a small peak at low noise level, going through a maximum at a medium noise level and decreasing again at a high noise level. Thus SR is observed in this model, it is plotted in Fig. 3. 


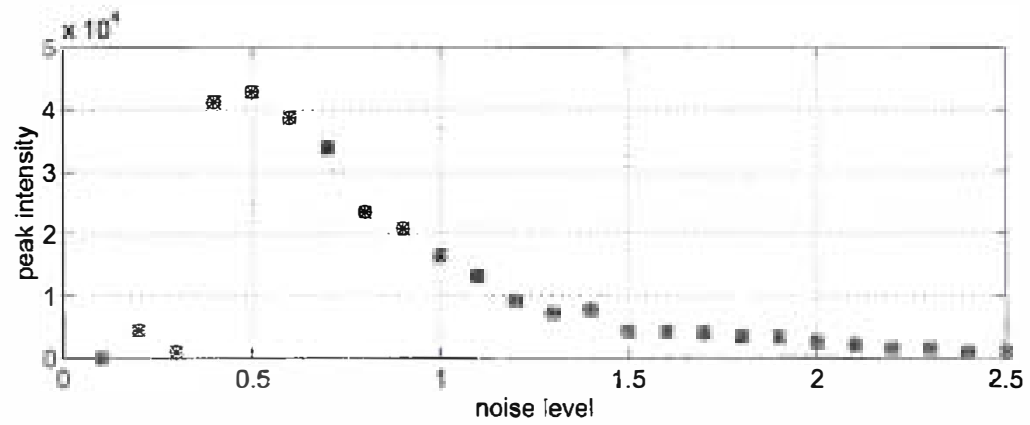

Iigure 3: 'The peak intensity (vertical axis) in the ISIH with the same period as the stimulus when the noise level $\tau_{\text {roise }}$ (horizontal axis) is varied. At a specific noise level a maximum in the coherent output occurs: this is the point of SR. Parameters $\theta=4, \gamma=0.0 \overline{5}, \tau_{\text {sig }}=2, \epsilon_{\text {sig }}=0.7$ and $\epsilon_{\text {noise }}=1$.

\section{Discussion}

We have seen that the simple model we used is capable of describing multimodal firing patterns and SR. The reason to choose this model lies in its simplicity. Several other models, that may seem more realistic in a biological sense, are that complex that finding an analytical description for the isIH turns out to be very difficuit, if not impossible. Although we haven't been able to write down such an expression for our model yet, it looks as if we are niaking progress.

\section{References}

Douglass, J. K., Wilkens, L., Pantazelou, E., and Moss, F. (1993). Noise enhancement of information transfer in crayfish mechanoreceptors by stochastic resonance.

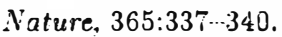

Gerstein, G. L. and Kiang, N. Y.-S. (1960). An approach to the quantitative analysis of electrophysiological data from single neurons. Biophysical Journal, 1:15- 28.

Gerstein, G. L. and Mandelbrot, B. (1964). Randow walk models for the spike activity of a single neuron. Biophysical Journal, 4:41-68.

Longtin. A. (1993). Stochastic resonance in neuron models. Journal of Statistical Physics, 70:309-327.

Mountcastle. V. B., Ta!kot, W. H., Sakata, H., and Hyvärinen, J. (1969). Cortical neuronal mechanisms in fiutter-vibration studied in unanesthetized monkeys neuronai periodicity and frequency discrimination. Neurophysiological journal, $32: 452-484$.

Pyke, R. (1959). The suprenum and infimum of the poisson process. Ann. Math. Statist.. 30:568-576.

Siegel, R. M. (1990). Non-linear dynamical system theory and primary visual cortical processing. Physica $D, 42: 385-395$. 